\title{
UTILIZATION OF THE THERMOELECTRIC SENSOR TO REALIZE A NEW FLUID LEVEL DETECTOR
}

\author{
K. HACHAMI ${ }^{\mathrm{a}}$, D. MOUSSAID ${ }^{\mathrm{a}, *}$, M. RAHMOUN ${ }^{\mathrm{b}}$ \\ and E. BENDADA ${ }^{b}$ \\ ${ }^{\mathrm{a}}$ Electronics and System Laboratory, Department of Physics, University Med $1^{\mathrm{er}}$. \\ Faculty of Sciences, Oujda Morocco \\ ${ }^{\mathrm{b}}$ Electronics, Instrumentation and Physics Measurement Laboratory, \\ Department of Physics, University My Ismail, Faculty of Sciences and \\ Technology, Errachidia Morocco
}

(Received 12 April 2001; In final form 15 May 2001)

\begin{abstract}
This paper describes a prototype thermoelectric sensor system which makes use of the thermoelectrical effects. The sensor includes two printed circuits, the first is constituted of a resistive constantan track, the second is a planar thermoelectric circuit constituted of many plated differential thermocouples. The first circuit is placed at the top of the second circuit so that the resistive tack is placed on the top of the even thermocouple junctions. The measuring method consists to passing an electrical current through the constantan track in order to generate temperature gradients between the junction points of the second circuit. Then the resulting temperature differences between the junction points is directly converted into a proportional Seebeck voltage. As an application, the sensor is placed into a reservoir and adapted in order to realize a fluid level detector.
\end{abstract}

Keywords: Level detector; Thermoelectric sensor; Resistive track; Plated thermocouples

\section{INTRODUCTION}

The hot wire anemometer has been used for many years as a research tool in fluid mechanics [1]. The Seebeck effect was implemented for long time to conceive and realize a great diversity of sensors, the measurement of physical size is a difference in temperature between

*Corresponding author. 
two points of a thermocouple [2,3]. The problem arising from the use of the traditional thermoelectric circuits are to solve the practical problems posed by the welding of many bimetallic contacts. The fabricating method of bimetallic circuits consists to deposit by electrolytic way a metal layer of high conductivity on a metal support of low conductivity $[4,5,6]$. In a previous works $[7,8]$, we described and characterized a new thermoelectric sensors in a shape of printed circuit, adapted to fluid flow measurement and a differential circuit breaker. The present work is an experimental study of the coupling between a resistive track circuit and other bimetallic circuit. The two circuits are placed one on the top of the other thus constituting a new thermoelectric sensor. As an application, the sensor is adapted and placed into a reservoir in order to realize a fluid level detector.

\section{DESCRIPTION}

The sensor includes two printed circuits maintained interdependent on Kapton support of $25 \mu \mathrm{m}$ thickness realized according to the famous engraving technique largely used in the printed circuit technology. The first circuit as shown in Figure 1, is a resistive constantan track with a thickness of $25 \mu \mathrm{m}$ and width of $0.4 \mu \mathrm{m}$. The electric resistance $R$ of constantan, with low temperature coefficient, is intended to be traversed by an electrical current $I$. The second circuit as shown in Figure 2 is a thermoelectric track comprising a continuous ribbon of constantan of thickness $25 \mu \mathrm{m}$ available on Kapton of thickness $25 \mu \mathrm{m}$. The ribbon is covered uniformly with an electrolytic copper deposit of high conductivity of thickness $5 \mu \mathrm{m}$. Using the photoengraving techniques, the circuit is engraved with iron perchloride following the form and the dimension desired. The ammonium per-

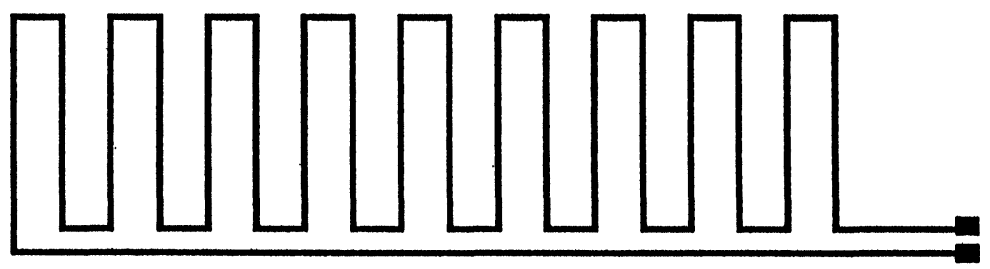

FIGURE 1 The constantan resistive track. 


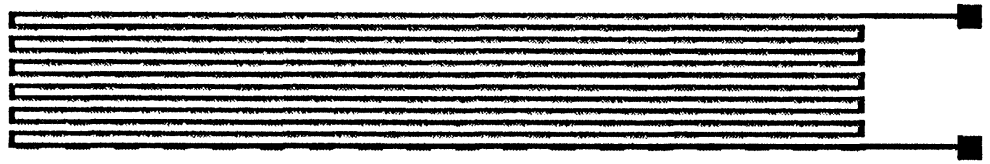

FIGURE 2 The bimetallic circuit.

xodisulfate attacks mainly copper and leaves intact the constantan sheet, is used to reveal the shape of the electrodes as shown in Figure 3 and the final form of the bimetallic circuit, shown in Figure 2, is comprised by many plated differential thermocouples interconnected in series. The expression of the output voltage delivered by an thermoelectric chain (Fig. 4) is only simple in the classic configurations where a great number of conductors of different nature are interconnected in series. Given that a bimetallic chain with plated electrodes is not made with homogenous conductors, it is necessary to determine the relationship voltage and temperature differences by going back to the classical theory of thermoelectricity. In order to
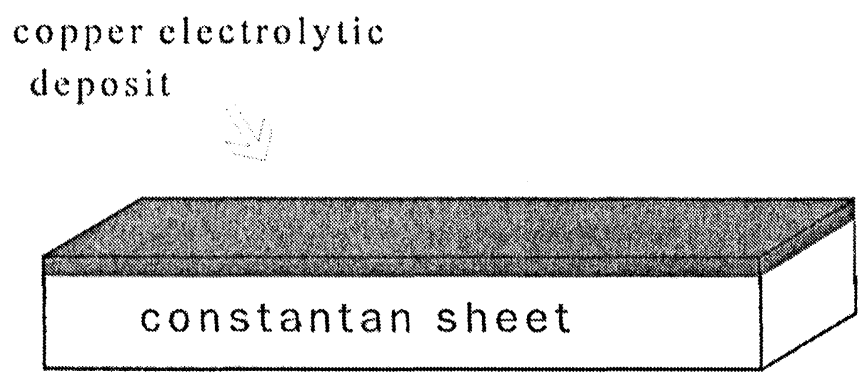

regular thermoclectric chain realized

by photoengraving technique

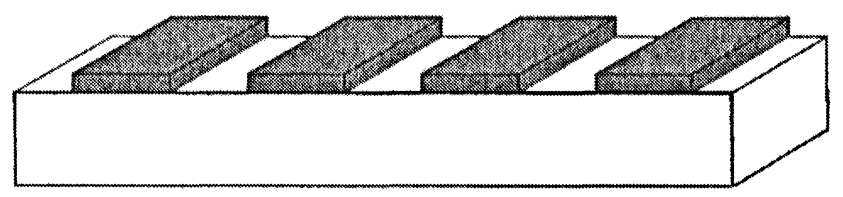

FIGURE 3 Thermoelectric chain. 


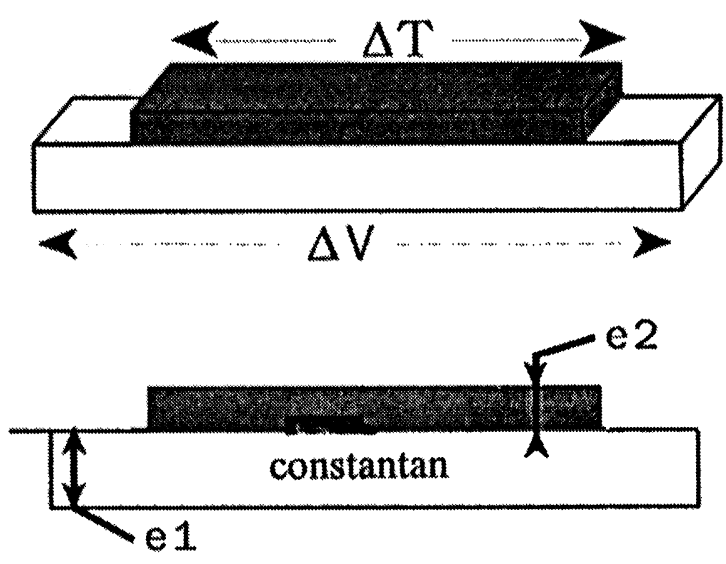

FIGURE 4 Thermoelectric element.

determine the expression of voltage delivered by Seebeck effect, is necessary to determine the equivalent thermoelectric capacity expression of a bimetallic circuit.

The constantan thermoelectric capacity is $\alpha_{1}$ (region not plated of copper), in the plated region of copper the equivalent thermoelectric capacity is given by:

$$
\alpha_{e q}=\frac{\alpha_{1} \sigma_{1} e_{1}+\alpha_{2} \sigma_{2} e_{2}}{\sigma_{1} e_{1}+\sigma_{2} e_{2}}
$$

where $\alpha_{i}$ is the thermoelectric capacity; $\sigma_{i}$ is the electric conductivity. In the plated region of copper, the equivalent thermoelectric capacity $\alpha_{e q}$ approach the thermoelectric capacity of copper and the temperature difference between two junction points is directly converted into a proportional Seebeck voltage: $\Delta V=\left(\alpha_{1}-\alpha_{e q}\right) \Delta T$.

In order to realize a thermoelectric sensor, the first circuit (Fig. 1) is placed on the top of the circuit (Fig. 2) so that the resistive track is placed on the top of the even junctions as shown in Figure 6. When the resistive track is crossed by an electrical current, generates temperature gradients by Joule effect on the even junction points as shown in Figure 5. This temperature gradients are proportional to the dissipated Joule power $P$ in the resistive track and the Seebeck output voltage 


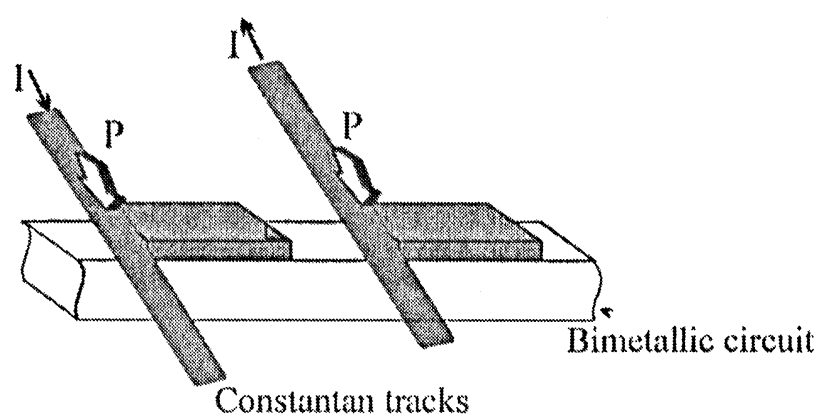

FIGURE 5 Section of the sensor.

generated by the bimetallic circuit is proportional to the power $P$ :

$$
\Delta V=\alpha_{G} \frac{P}{\left[\operatorname{coth}(\gamma l / 2) / R_{c}\right]+\left[\operatorname{coth}\left(\gamma_{u} l_{u} / 2\right) / R_{c_{u}}\right]}
$$

with $\alpha_{G}$ : coefficient dependent of the thermoelectric capacity, the electric conductivity of the couple copper-constantan and their dimensions. $R_{u}, R_{c u}$ : resistance characteristic of region with copper and region with no copper. $l, l_{u}$ : length of cooper region and region with no copper. $\gamma$ : heat propagation constant.

\section{APPLICATION FOR MEASURING A FLUID LEVEL IN A RESERVOIR}

The measurement of a fluid level in a reservoir presents a big industrial interest. For this application, the sensor is realized with two

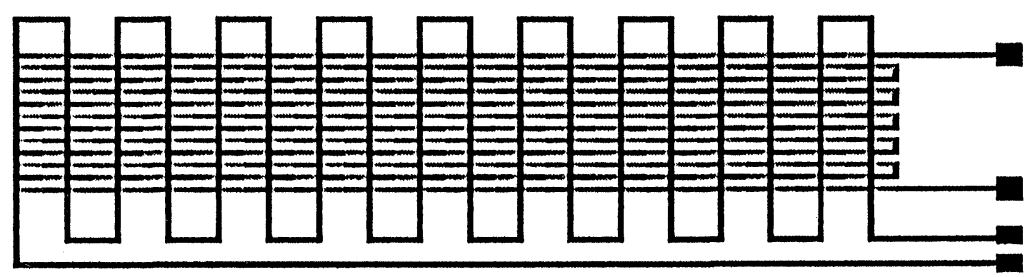

FIGURE 6 Used sensor. 
circuits one on the top of the other as shown in Figure 6. The resistive track is placed on the top of the even junctions of the bimetallic circuit of length $280 \mathrm{~mm}$ (folded up as shown in Fig. 2), width $0.4 \mathrm{~mm}$, space between plated electrodes is $4 \mathrm{~mm}$ and the electric resistance $41.6 \Omega$.

\subsection{Measurement of Thermal Gradients Caused by the Electrical Current}

When the resistive track is crossed by an electrical current, the temperature gradients generated by Joule effect are localized on the even junction points. The temperature differences are directly converted into a proportional Seebeck voltage. The variation of this voltage delivered by the bimetallic circuit as a function of current is shown in Figure 7 for the used fluid (water). It is noted that the Seebeck voltage is proportional to the dissipated Joule power in the resistive track. The value $(60 \mathrm{~mA})$ of the electrical current has been chosen in order to have a sufficient graduating.

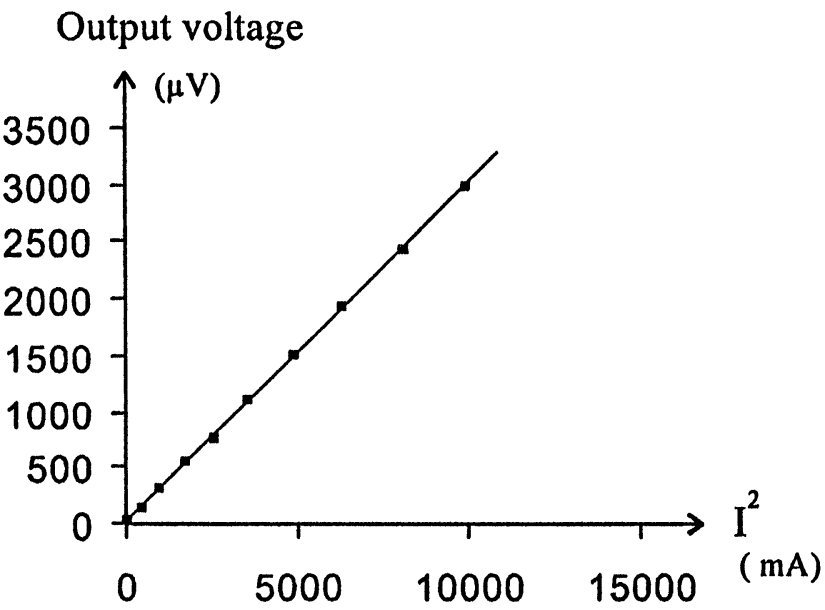

FIGURE 7 Variation of the output voltage against the current square. 


\subsection{Fluid Level Detector}

In order to realize a fluid level detector, the sensor is partially immersed into a reservoir, containing a fluid, and the sensor is glued on the side of the reservoir as shown in Figure 8.

The low value of electrical current doesn't create a particular problem of security (the constantan track of resistance $22 \Omega$ is crossed by an electrical current of $60 \mathrm{~mA}$, generates a power of $80 \mathrm{~mW}$ ). The experimental results shows that the output voltage $(541 \mu \mathrm{V})$ detected, when the sensor is completely immersed in the fluid, is lower than the output voltage $(1100 \mu \mathrm{V})$ when the sensor is out.

The experimental results enable us to trace a variation of the output voltage delivered by the sensor against a fluid level in a reservoir as shown in Figure 9 and the calibration curve is linear. The knowledge of the curve slope $S=\Delta V / \Delta h=8.42 \mu \mathrm{V} / \mathrm{mm}$ permits to determine the fluid level in the reservoir according the relationship: $h=h_{0}+\Delta V / S$, where $h_{0}$ : level where the sensor is placed in relation to the reservoir bottom. It is noted that the output voltage varies with the fluid level in the reservoir, therefore the sensor can be used as a fluid level detector.

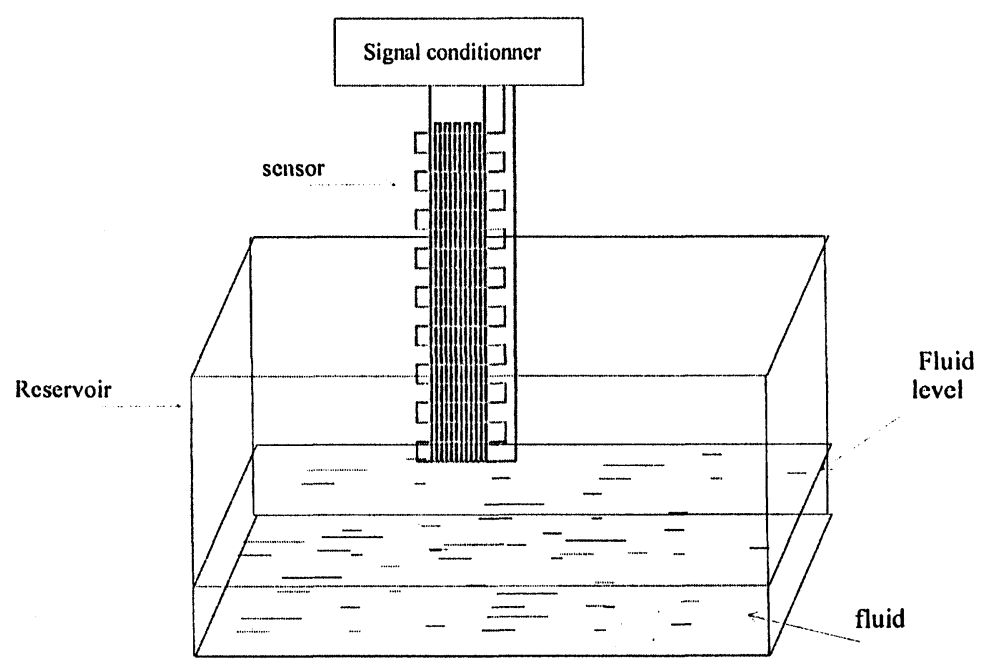

FIGURE 8 Device constituting the level detector. 


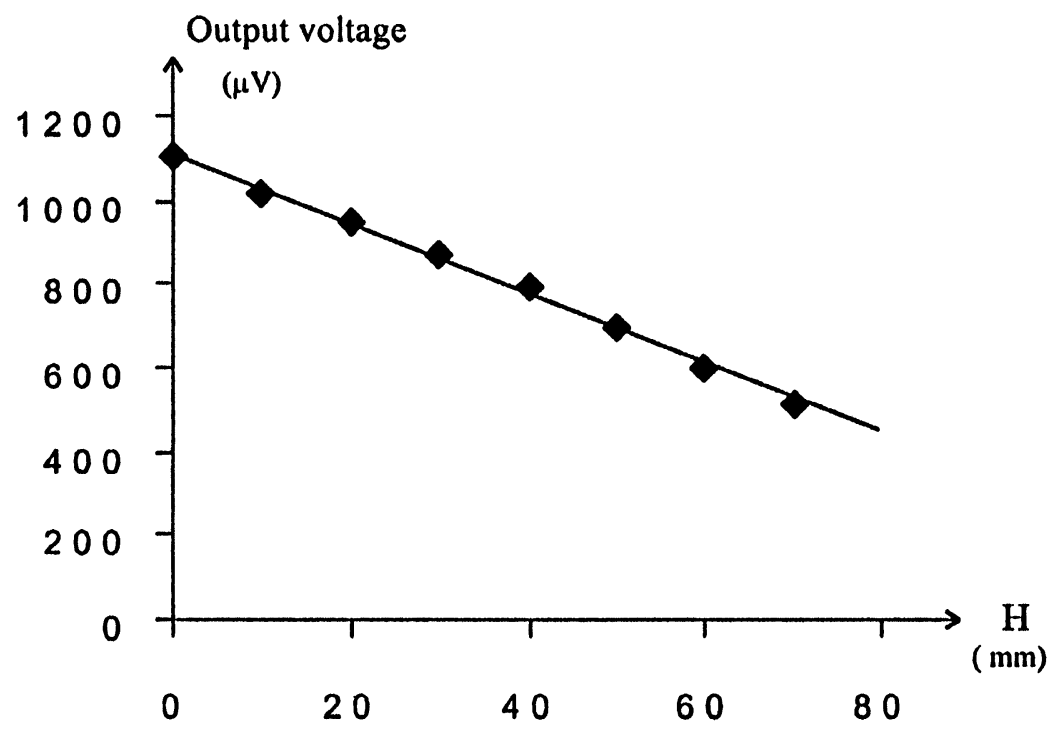

FIGURE 9 Variation of the output voltage against the fluid level.

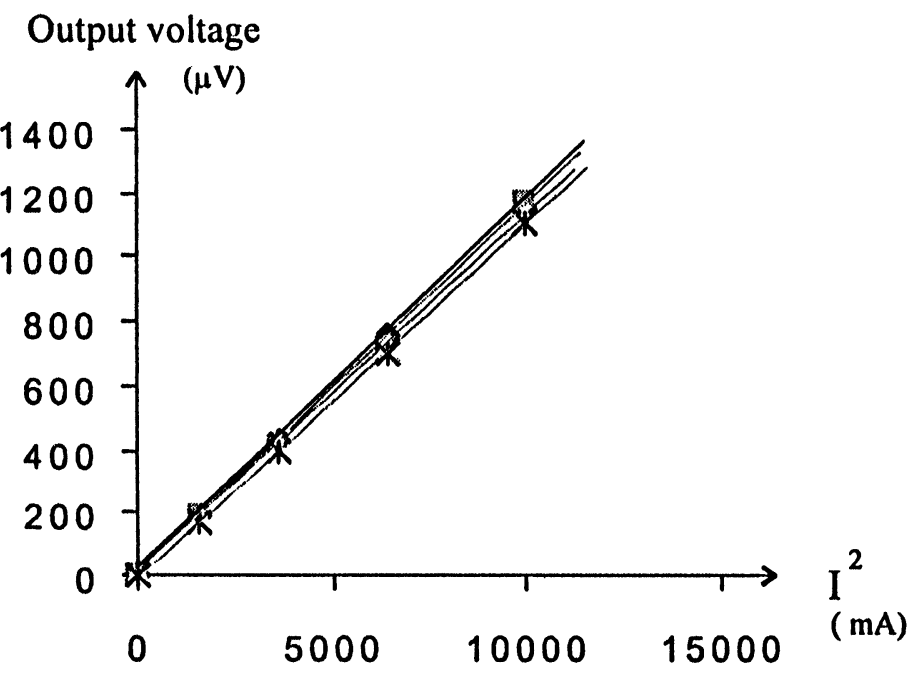

FIGURE 10 Variation of the output voltage against the current square for different temperatures $T=0^{\circ} \mathrm{C} ; 17^{\circ} \mathrm{C} ; 30.5^{\circ} \mathrm{C}$ and $43^{\circ} \mathrm{C}$. 


\subsection{Specific Temperature Effect}

Since positive and negative heat losses are generated around the average temperature of the sensor which is equal to that of the fluid, there must be no change in the output voltage when the sensor is immersed in a fluid whose temperature varies. The experimental results permits to trace the output voltage variation as a function of the electrical current for different fluid temperatures as shown in Figure 10. The calibration curve shows that the temperature dependence of the output voltage is small with the relative variation $1 / S$ $d S / d T=3.5 \cdot 10^{-3}$. Then, temperature variation of $3^{\circ}$ could be interpreted like 1/100 level variation. The simplest approach for temperature compensation consists in associating another same sensor. This second sensor is completely immersed in the fluid as shown in Figure 11. When compensating the temperature dependence of the sensor it was observed the previous calibration curve is not dependent on fluid temperature in the range $0-43^{\circ} \mathrm{C}$.

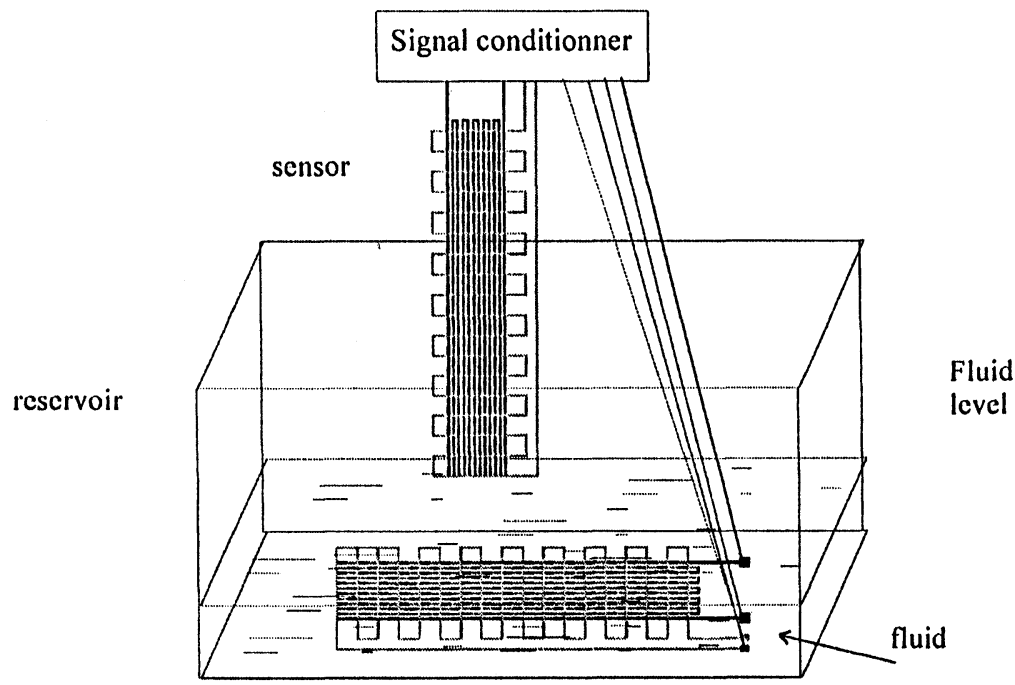

FIGURE 11 Device permitting temperature correction. 


\section{CONCLUSION}

This study demonstrates that the resistive track placed on the top of the bimetallic circuit enabled us to realize a new fluid level detector. This sensor is sensitive and can be applied for many industrial applications. As an application, the sensor can be adapted to detect the temperature radiation.

\section{References}

[1] M. M. Bruun, M. A. Kahn, H. I. Kaijien and A. A. Farad (1988) Velocity calibration for hot wire anemometry. J. Phys. E. Sci. Instr. Vol. 2, 225-232.

[2] R. L. Weber (1941) Heat and Temperature. Ann. Inst. Physiques, Temperature, its measurement and control in science and industry (Practice-Hall, Englewood cliffs).

[3] T. Kwikketrs (1988) Two thick film sensors. Hybrid circuit 16, 32-42.

[4] P. H. Herin and P. Thery (1992) Measurement on the thermoelectric properties of thin sensors. Measurements Sciences and Techology Vol. 3, 495-500.

[5] P. Thery and J. Pauquet (1980) Propriétés thermoélectriques des systèmes à deux couches conductrices superposées. J. of Physiques E Sci. Instruments 13, 323-327.

[6] D. Leclerq, F. Wattiau and P. Thery Procédé et dispositif utilisant les effets thermoélectriques pour la mesure d'unc grandeur physique dont la variation est apte à modifier les propriétés thermophysiques d'un milieu. French Patent 8916809.

[7] M. Rahmoun, A. El Hassani, D. Leclerq and E. Bendada (1999) Peltier effect applied to the design and realization of a new mass flow sensor. J. Active and Passive Elec. Comp. Vol. 22, 165-174.

[8] K. Hachami, D. Moussaid, M. Rahmoun and A. El Hassani (2000) Design and realization a new thermoelectric sensor. Application Active and Passive Elec. Comp. Vol. 23, 145-155. 

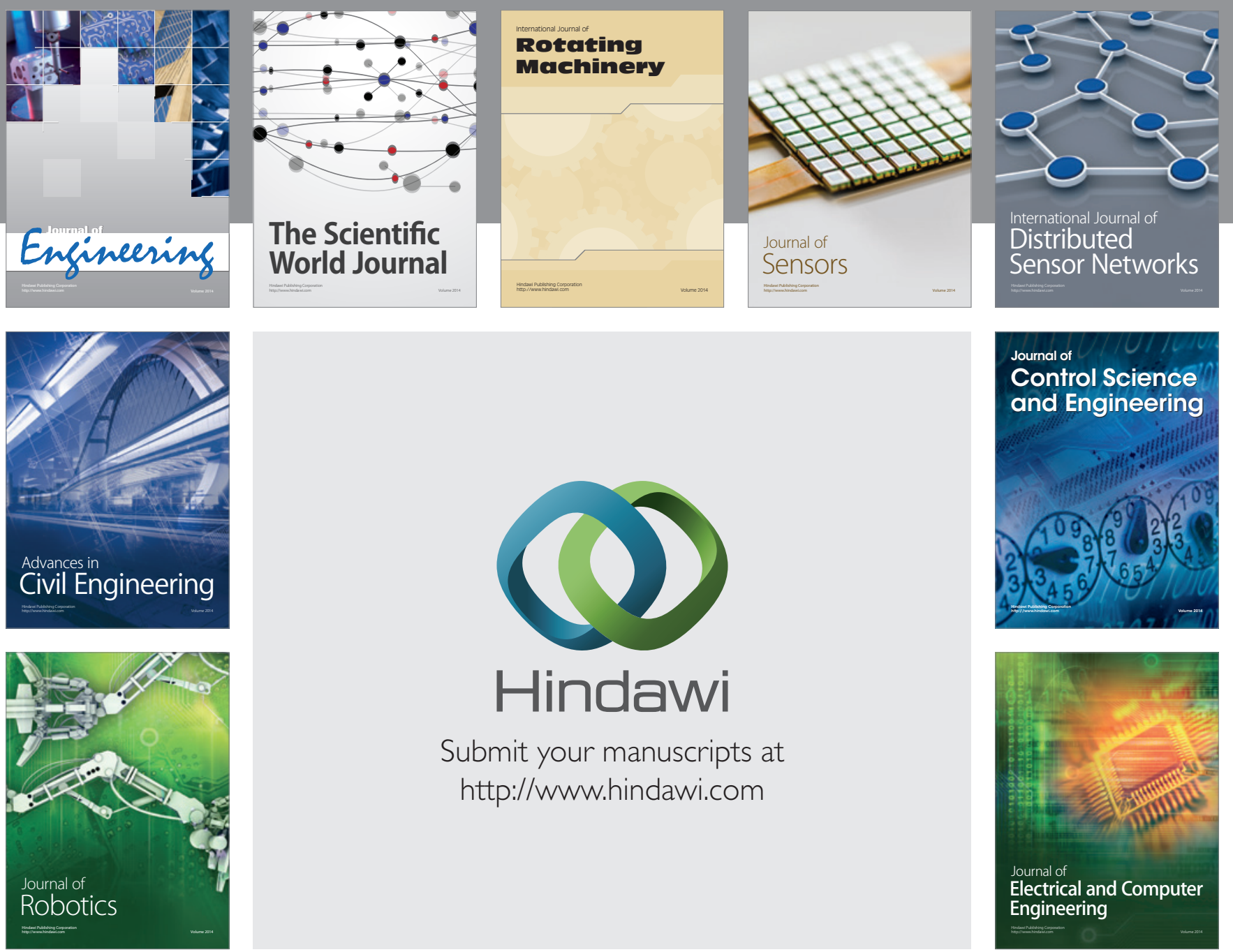

Submit your manuscripts at

http://www.hindawi.com
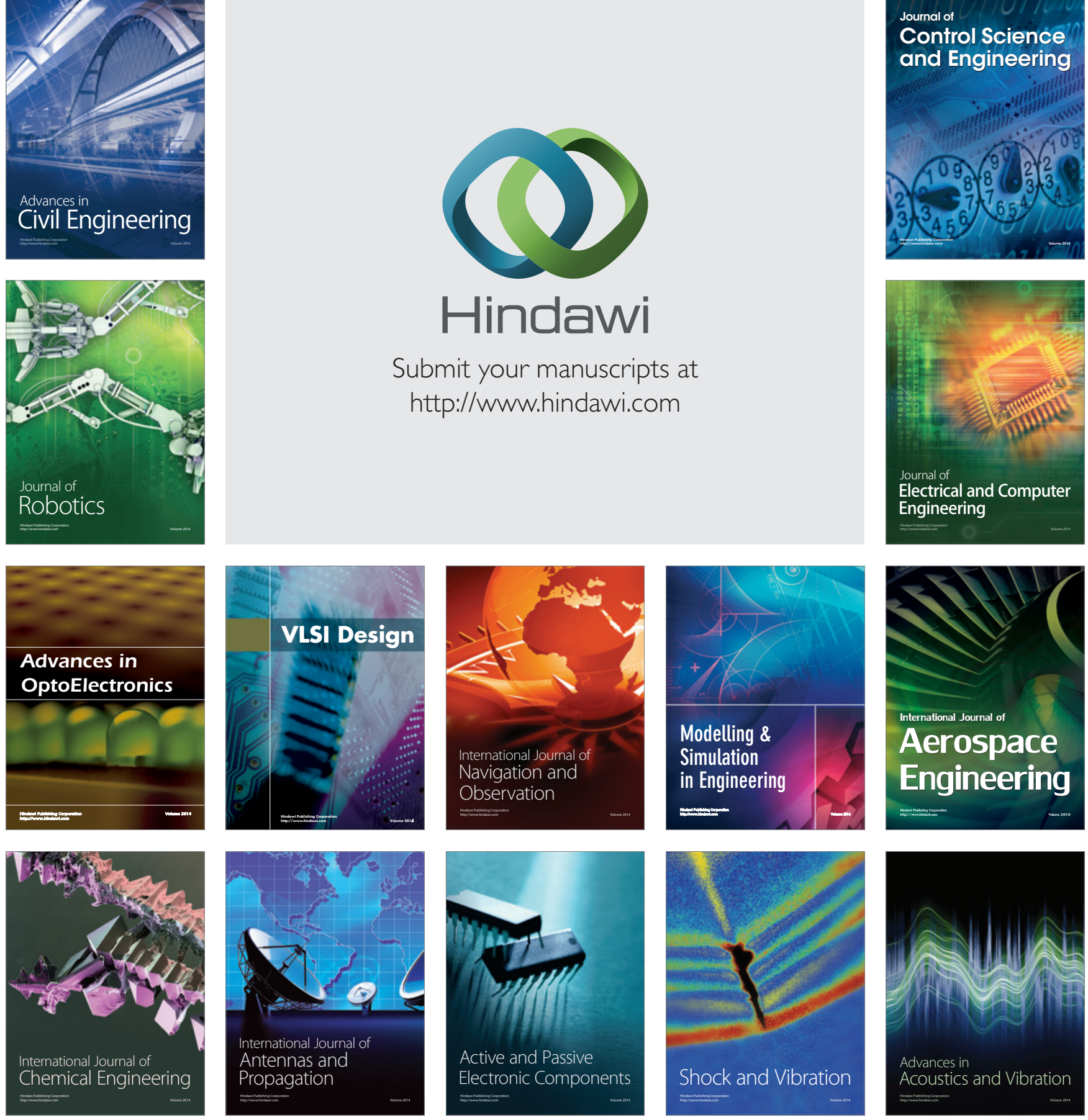